\title{
Evaluation of proteomics responses of rice (Oryza sativa) during arsenic toxicity amelioration by a potential microbial consortium
}

\author{
S. Awasthi ${ }^{1}$, R. Chauhan ${ }^{1}$, S. Srivastava ${ }^{2}$ \& R.D. Tripathi ${ }^{1}$ \\ ${ }^{1}$ CSIR - National Botanical Research Institute, Varanasi, Uttar Pradesh, India \\ ${ }^{2}$ Institute of Environment \& Sustainable Development, Banaras Hindu University, Varanasi, Uttar Pradesh, India
}

\begin{abstract}
Environmental pollution of arsenic (As) is a serious problem that is not only affecting the local people but also population throughout the world through rice and rice-based food products. Rice accumulates As in significantly greater amounts in comparison to other cereal crops. Microbiological interventions can serve as protective agents to ameliorate As stress in rice. The experiments were conducted in hydroponically grown in rice seedlings subjected to arsenate $[\mathrm{As}(\mathrm{V})]$ stress for $15 \mathrm{~d}$. Two-dimensional gel electrophoresis analysis of 340 spots revealed that 145 spots were differentially expressed; out of these, 87 spots were identified using MALDI-TOF-TOF. The differentially expressed proteins during supplementation of microbial consortium under As stress belonged to defense, growth promotion, energy metabolism, cellular and metabolic processes.
\end{abstract}

\section{INTRODUCTION}

Arsenic (As) exposure from rice consumption has now become a global health issue. Rice (Oryza sativa L.), a staple food in many Asian countries is a crop with high accumulation of As, which are highly toxic and nonessential elements for human body. Arsenic is initially absorbed from the soil through the roots and enters the shoot via the xylem and from the xylem of stem it is transported to the phloem and finally into the grain. Microbes that are able to grow in As contaminated environment are equipped with genetic architecture, metabolic pathways and enzymatic systems that enable them either to tolerate As toxicity via transformation of toxic inorganic forms into less toxic organic ones or to use As as a part of metabolism. Proteins are functionally versatile macromolecules that play major role in living cells. In the present study, a comparative proteomics analysis coupled with photosynthesis and antioxidant enzymes analysis on rice plants subjected to As stress has been adopted, to identify the protein profile associated with consortium of Pseudomonas putida MTCC5279 (P. putida) and Chlorella vulgaris induced resistance to As stress and to explore the possible molecular mechanisms involved.

\section{METHODS}

\subsection{Plant material and experimental conditions}

Oryza sativa L. var. Triguna was used for the experiments. Plants were exposed to different treatments $[50 \mu \mathrm{M} \mathrm{As}(\mathrm{V})]$, P. putida and C. vulgaris and coinoculation of P. putida and C. vulgaris for $15 \mathrm{~d}$. After
15 days exposure, 3 replicates were harvested for proteome analysis, expression of the selected genes and As accumulation.

\subsection{Protein extraction, 2DE analysis, image analysis, protein identification and MALDI-TOF-TOF analysis}

Shoot tissue $(5 \mathrm{~g})$ was pulverized to a fine powder in liquid nitrogen and suspended in $10 \mathrm{~mL}$ lysis buffer. Suspension was mixed with Tris saturated phenol $(\mathrm{pH}$ 8.0) for $30 \mathrm{~min}$ with gentle shaking and centrifuge at $9000 \times \mathrm{g}$ for $10 \mathrm{~min}$. After that tissue was precipitated in $0.1 \mathrm{M}$ ammonium acetate for overnight at $-200^{\circ} \mathrm{C}$. Again centrifuged and the dried pellet was dissolved into rehydration buffer. Total shoot protein was determined by Bradford assay (Bio-Rad, USA). Isoelectric focusing was carried out with $250 \mu \mathrm{g}$ of protein as given by Agrawal et al. (2008). The strips were then loaded on top of $12.5 \%$ poly acrylamide gels for SDS-PAGE. The gels were fixed and stained with a silver stain plus kit as per protocol (Bio-Rad, USA). The silver stained gel obtained after 2DE were scanned for image acquisition using the Bio-Rad Fluor $\mathrm{S}$ system equipped with a 12-bit camera. Images from three biological replicate 2-DE gels were taken for the data analysis in PD Quest version 8.0.1 (Bio$\mathrm{Rad})$. The silver stained protein spots were digested for MALDI MS/MS were digested with trypsin (Promega Corporation, MA, USA) as per manufacturer instruction. The stained protein spots were excised manually, washed thoroughly with water and crushed in small pieces and digested with trypsin. Peptide solution mixed with $5 \mathrm{mg} \mathrm{mL}^{-1}$ CHCA (a-Cyano-4-hydroxy 


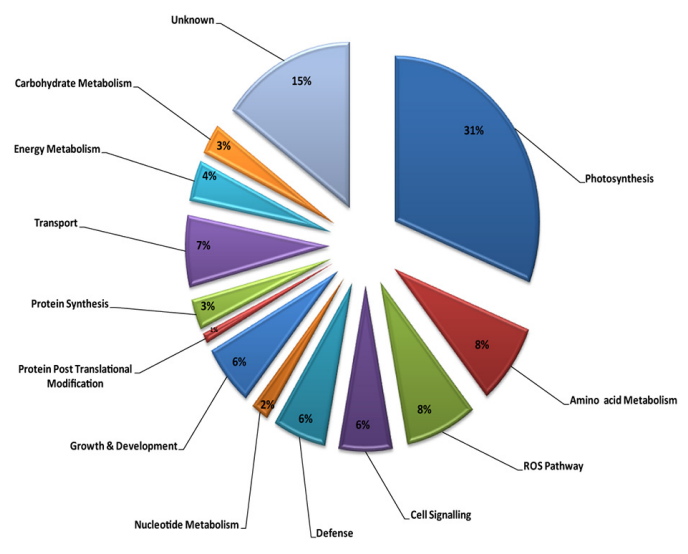

Figure 1. Classification of identified differentially expressed rice shoot proteins in various functional classes based on their putative functions assigned them using protein database.

cinnamic acid) matrix and spotted on to MALDI plate. A 4800 Proteomics Analyzer (Applied Biosystems, USA) with TOF/TOF optics was used for all MALDI-MS and MS/MS applications. In all the protein identifications, probability scores were greater than the score fixed by Mascot as significant with a $\mathrm{p}<0.05$.

\section{RESULTS AND DISCUSSION}

\subsection{Identification of differentially abundant proteins in rice}

The present study indicates a practical potential for microbial application as an intervention strategy in mitigating As toxicity and reducing As uptake and translocation in rice plants. Supplementation of $P$. putida and C. vulgaris with $50 \mu \mathrm{M} \mathrm{As}(\mathrm{V})$ significantly alleviated As induced growth inhibition in rice seedlings (Fig. 1), improved photosynthetic and antioxidant machinery and significantly decreased rice As concentration. Many proteins were differentially induced in rice seedlings by $P$. putida and $C$. vulgaris under As stress. The differentially expressed proteins were mainly involved to regulate defense system, photosynthesis, detoxification, plant hormones and energy metabolism. Heat shock protein are also found to be accumulated, which were a class of functionally related proteins involved in repair and aid in the renaturation of stress-damaged proteins, and reestablish cellular homeostasis, protect cells against the damage of stress (Ahsan et al., 2009). This study also revealed the expression of IAP100 (Inhibitor of Apoptosis) in the presence of consortium under As stress; IAP100 has ability to regulate caspases and also influence ubiquitin (Ub)-dependent pathways that modulate innate immune signaling. In conclusion, results provide new insights about the proteins and mechanisms involved in microbial consortium mediated As toxicity amelioration in rice.

\section{CONCLUSIONS}

The comparative proteomic analyses of rice leaf revealed that there were several complex metabolic interactions leading to consortium mediated resistance against As stress. The present study provides important protein profile on the basis of consortium mediated improved resistance against As stress in rice. The identified proteins would be helpful for better understanding the complex response network under As stress and may open new avenues for seeking new strategies for As detoxification in rice plants.

\section{ACKNOWLEDGEMENT}

Surabhi Awasthi is thankful to Council of Scientific \& Industrial Research for the award of Senior Research Fellow.

\section{REFERENCES}

Agrawal, L. Chakraborty, S. Jaiswal, D. K. Gupta, S. Datta, A. \& Chakraborty, N. 2008. Comparative proteomics of tuber induction, development and maturation reveal the complexity of tuberization process in potato (Solanum tuberosum L.). J. Proteome Res. 7(9): 3803-3817.

Ahsan, N. Renaut, J. \& Komatsu, S. 2009. Recent developments in the application of proteomics to the analysis of plant responses to heavy metals. Proteomics 9(10): 2602-2621. 